\title{
Successful Treatment of Refractory Gout Using Combined Therapy Consisting of Febuxostat and Allopurinol in a Patient with Chronic Renal Failure
}

\author{
Michitaka Maekawa, Hidetaka Tomida, Takafumi Aoki, Manabu Hishida, \\ Takatoshi Morinaga and Hirofumi Tamai
}

\begin{abstract}
Gouty arthritis is a metabolic disorder associated with hyperuricemia. Despite the development of novel pharmacotherapies, some hyperuricemia patients are drug refractory and develop gout. A 74-year-old man with frequent gouty attacks and chronic renal failure presented with asymmetrical polyarthritis affecting multiple joints. The diagnosis of gout was confirmed based on the presence of monosodium urate crystals in the patient's right wrist. The administration of systemic corticosteroids relieved the joint inflammation and pain; however, the urate level increased to $28 \mathrm{mg} / \mathrm{dL}$ and the gout attacks recurred. Combined allopurinol, febuxostat, and benzbromarone therapy reduced the urate level to $<6 \mathrm{mg} / \mathrm{dL}$, and the attacks gradually declined. This is the first report of two xanthine oxidase inhibitors being used to treat refractory gout.
\end{abstract}

Key words: febuxostat, allopurinol, benzbromarone, combination therapy, renal failure, gout

(Intern Med 53: 609-612, 2014)

(DOI: 10.2169/internalmedicine.53.0698)

\section{Introduction}

Gout is a debilitating condition caused by the deposition of monosodium urate crystals in and around joint tissue. The incidence of gout increases in association with the degree of elevated serum urate (hyperuricemia) (1). Maintaining a serum urate level of $<6 \mathrm{mg} / \mathrm{dL}$ is recommended for reducing the incidence of acute attacks in patients with a history of gout (2). A decreased kidney function leads to hyperuricemia. The management of gout in patients with renal impairment is challenging because the hyperuricemia is resistant to uricosuric drugs, such as benzbromarone, and the patients are also at greater risk of side effects (3). In addition, while xanthine oxidase inhibitors (XOIs), such as allopurinol, are effective against gout in many patients, the dose must be reduced in patients with renal impairment (3). Febuxostat is a recently introduced XOI that has been shown to be effective and safe in many patients with renal impairment and gouty attacks. However, recent clinical trials have reported that even high doses of febuxostat are insufficient to manage hy- peruricemia in a significant proportion of renal-deficient gout patients. It is unclear how best to manage hyperuricemia in gout patients with functional kidney impairment when standard therapy with a single XOI is insufficient.

We herein report the case of a patient with refractory gout that was successfully treated by combining two different XOIs, namely allopurinol and febuxostat.

\section{Case Report}

A 74-year-old Japanese man with a longstanding history of hypertension and chronic renal failure was admitted to our hospital due to polyarthritis that had persisted for several days. He was on loop diuretics for hypertension and low-dose aspirin to prevent the recurrence of cerebral infarction. Initially, his right hand was swollen; however, the swelling then spread to both hands, shoulders, hips, knees and ankles over the next few days, with rapidly increasing severity. There were no subcutaneous nodules around the patient's joints or auricles. Radiographs of his hands showed soft tissue swelling without areas of bony erosions. His esti- 


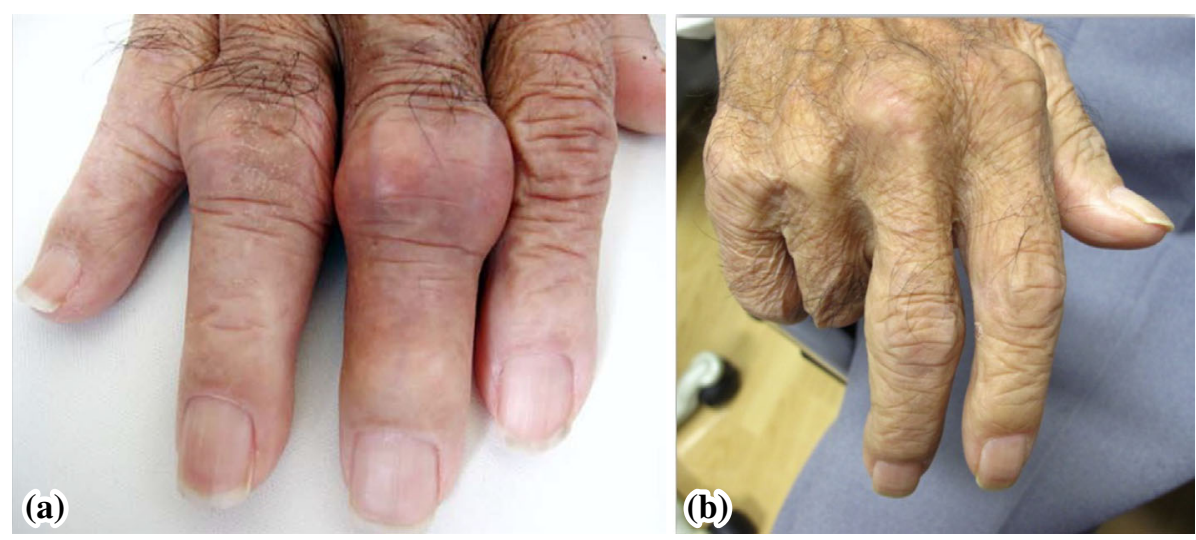

Figure 1. Tophi over the interphalangeal joints in the patient's left hand. (a) Before therapy with febuxostat. (b) After three months of combination therapy.

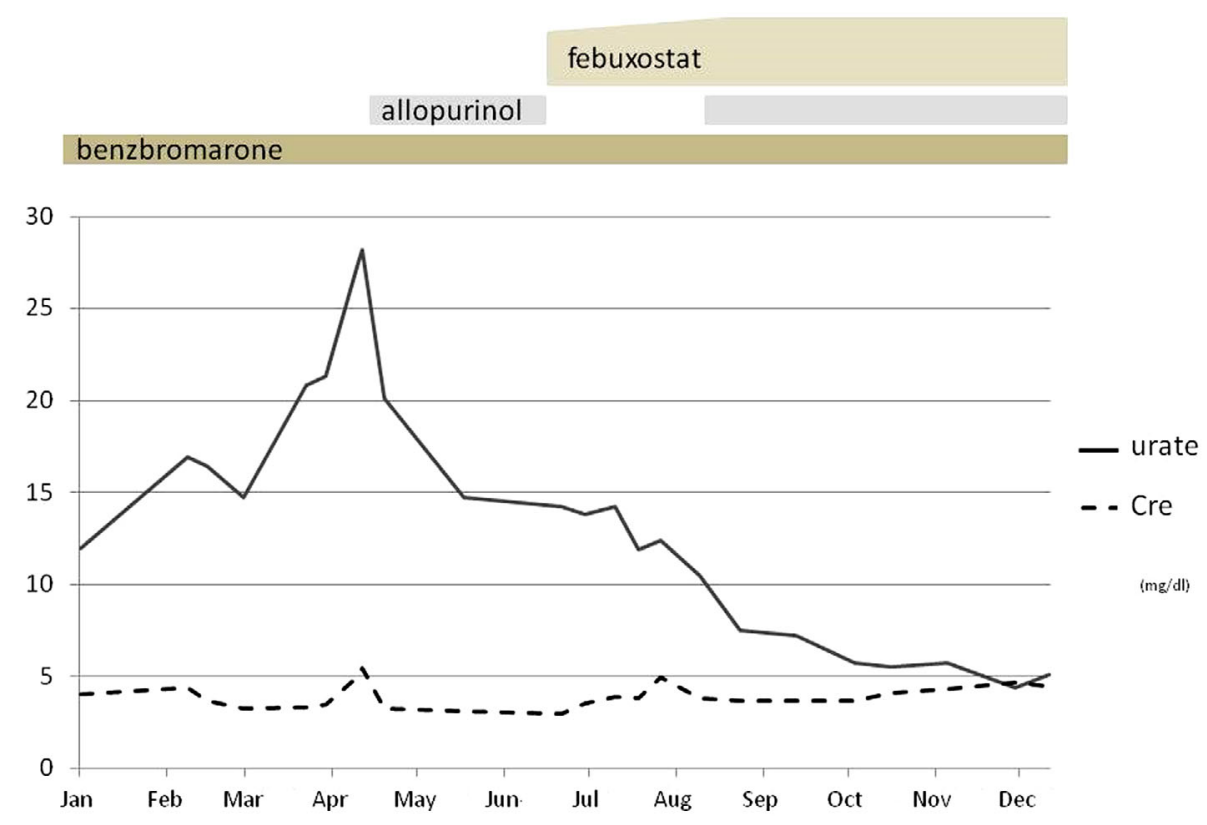

Figure 2. Course of the urate level in relation to the administration of urate-lowering therapy. The uric acid levels declined below $6 \mathrm{mg} / \mathrm{dL}$ following combination treatment with febuxostat, allopurinol and benzbromarone.

mated glomerular filtration rate (eGFR) was $13.7 \mathrm{~mL} / \mathrm{min}$ per $1.73 \mathrm{~m}^{2}$. The serum urate level $(16.4 \mathrm{mg} / \mathrm{dL})$, elevated CRP level (20.2 mg/dL) and presence of leukocytosis $(13,200 / \mu \mathrm{L})$ were consistent with a clinical diagnosis of gout. Monosodium urate crystals were found in the synovial fluid aspirated from the patient's right wrist, confirming the diagnosis. A joint fluid culture was negative. Owing to an impaired kidney function, we did not administer nonsteroidal anti-inflammatory drugs (NSAIDs) and instead initiated treatment with oral prednisolone at a dose of $20 \mathrm{mg}$ per day, which resulted in rapid relief of the patient's arthritis symptoms.

Three weeks after the first gouty attack resolved, a second episode occurred accompanied by an extraordinarily high urate level of $28.2 \mathrm{mg} / \mathrm{dL}$. Tophaceous nodules appeared on the joints of the patient's fingers and toes during the attack (Fig. 1). The patient had experienced gouty attacks 30 years prior to the current episode; however, no gouty arthritis had developed, since he was on long-term benzbromarone treatment. We recognized the need for additional urate-lowering therapy and added allopurinol (50 mg/day) to benzbromarone. However, the urate level was approximately $10 \mathrm{mg}$ / $\mathrm{dL}$, which was higher than the clinical norm; therefore, we discontinued allopurinol and started combination therapy consisting of febuxostat with benzbromarone. Continued hyperuricemia led us to use all three urate-lowering agents, and combined treatment with allopurinol, febuxostat and benzbromarone lowered the uric acid levels to below $6 \mathrm{mg} /$ $\mathrm{dL}$, which was within the normal range (Fig. 2). We also used low-dose prednisolone (below $5 \mathrm{mg}$ per day) for six months as prophylaxis for acute gout. The acute attacks gradually became less frequent, and all tophi disappeared after three months of combined treatment. No obvious adverse events were reported during the combination therapy. 


\section{Discussion}

Our patient suffered from severe polyarthritis, not only in the peripheral joints (hands, toes and knees), but also in the cervical spine, shoulders and hip joints. The administration of three urate-lowering agents was required to achieve an optimal serum urate level in this frail elderly man (weight: $40 \mathrm{~kg}$ ) with chronic renal failure.

A decreased kidney function, loop diuretics and low-dose aspirin are all thought to contribute to the occurrence of elevated urate levels. Although it is uncertain why the patient's gout recurred after an interval of 30 years, the effect of a uricosuric agent (benzbromarone) appeared to diminish in association with a gradually deteriorating renal function, resulting in an elevated serum uric acid levels. Therefore, in addition to treatment with benzbromarone, the administration of a urate-lowering agent that inhibits urate production was a reasonable treatment option.

The accepted target level of serum urate in gout patients is below $6.0 \mathrm{mg} / \mathrm{dL}(3,4)$, a level that could not obtained in this case, even using treatment with febuxostat and benzbromarone combined at the maximum approved doses (for Japan).

Treating gout in patients with renal impairment is therapeutically challenging $(2,5)$. When treating acute gouty arthritis, the administration of colchicine and NSAIDs should be avoided due to the increased risk of side effects. Oral or intravenous corticosteroids are used to effectively treat acute attacks when colchicine and NSAIDs are ineffective or contraindicated; for example, in cases of moderate to severe renal insufficiency (4). Although there remains a risk of side effects, low-dose corticosteroids can be used for prophylaxis of acute gout attacks in patients with renal failure (3).

Medications for hyperuricemia include allopurinol, benzbromarone and probenecid. However, there is concern about the higher rate of adverse events associated with allopurinol in patients with a decreased kidney function. Hypersensitivity to allopurinol occurs in approximately $2 \%$ of patients, and patients with renal insufficiency have an even greater risk of developing this condition; therefore, the use of a limited dosage based on therenal function is recommended (4). Similar to uricosuric agents, XOIs are also less effective in reducing the uric acid levels in patients with a reduced renal function. One study found that none of the chronic kidney disease patients examined (with a serum creatinine level of $1.5-2 \mathrm{mg} / \mathrm{dL}$ ) reached the target urate level at a reduced dose of $100 \mathrm{mg}$ of allopurinol per day (6). Moreover, uricosuric agents are thought to be much less effective in patients with a glomerular filtration rates of $<50 \mathrm{~mL} / \mathrm{min}$ (4).

In the 1990s, the clinical emphasis shifted from lowering the serum uric acid level to providing analgesia for patients with chronic tophaceous gout owing to renal impairment because very few patients responded to drugs available at the time (7).

The introduction of the novel XOI febuxostat has proven beneficial for many of these patients, and the drug is widely prescribed for gout and hyperuricemia. In Europe and the United States, $80 \mathrm{mg}$ and $120 \mathrm{mg}$ tablets of febuxostat are available, respectively, while only $60 \mathrm{mg}$ per day is approved in Japan. Our patient did not respond to febuxostat alone, possibly due to the low dose prescribed for his lower body weight, and his hyperuricemia appeared to be resistant to febuxostat therapy. Recent clinical trials have revealed that even high-dose febuxostat therapy cannot achieve the target urate levels in some patients. For example, in the FACT trial, $24 \%$ of patients treated with $80 \mathrm{mg}$ per day of febuxostat did not achieve a serum uric acid level below 6 $\mathrm{mg} / \mathrm{dL}(8)$, while another study demonstrated that $6 \%$ of patients treated with febuxostat at a dose of $240 \mathrm{mg}$ per day did not achieve the target serum urate levels (6).

Another uricosuric agent, probenecid, is thought to have been ineffective in this case due to the patient's kidney function impairment. Moreover, some researchers have expressed concern regarding tubular damage caused by uric acid following the administration of uricosuric agents in chronic kidney patients (9). Therefore, we administered an additional XOI rather than treating the patient with a second uricosuric agent.

Controversy remains over how to best manage gout in patients refractory to a single urate-lowering agent (10). Some studies have demonstrated the efficacy of combining an XOI and uricosuric agent $(11,12)$, while others advocate the administration of high-dose febuxostat (up to $240 \mathrm{mg}$ ) and argue against the concurrent use of multiple XOIs due to concerns that the drugs would simply compete for the same active enzyme site (10). Despite such uncertainties, these two XOIs showed an additive effect, achieving the target range for the uric acid level in our patient. A recent study reported the efficacy of recombinant urate oxidase rasburicase for treating severe and refractory gout (13); however, this agent is expensive and not approved for the treatment of gout in many countries, including Japan.

Our case demonstrates the efficacy of combining two different XOIs, febuxostat and allopurinol. Combination therapy may be an effective and promising treatment option for patients with refractory gout and hyperuricemia. Although some studies have shown the efficacy and safety of febuxostat in patients with mild to moderate kidney dysfunction, there is a paucity of available data regarding the pharmacokinetics of this agent in patients with severe kidney dysfunction (14). In addition, there are presently no available pharmacokinetic studies of the co-administration of two different XOIs in patients with or without kidney dysfunction. More investigations of the safety profiles of febuxostat and combined therapy in patients with end-stage renal disease are required. Clinicians must consider the benefits and unknown risks associated with treatment before deciding there is adequate evidence of safety.

We herein demonstrated the efficacy of combined allopurinol and febuxostat in reducing the urate level and improving gout in an elderly patient with renal impairment. 
Further studies and clinical trials are needed to confirm the effectiveness and safety of this combination treatment regimen, especially in patients with renal failure.

The authors state that they have no Conflict of Interest (COI).

\section{References}

1. Compion EW, Glynn RJ, DeLabry LO. Asymptomatic hyperuricemia. Risks and consequences in the Normative Aging Study. Am J Med 82: 421-426, 1987.

2. Shoji A, Yamanaka H, Kamatani N. A retrospective study of the relationship between serum urate level and recurrent attacks of gouty arthritis: evidence for reduction of recurrent gouty arthritis with antihyperuricemic therapy. Arthritis Rheum 51: 321-325, 2004.

3. Gaffo AL, Saag KG. Management of hyperuricemia and gout in CKD. Am J Kidney Dis 52: 994-1009, 2008.

4. Terkeltaub RA. Clinical practice: gout. N Engl J Med 349: 1647$1655,2003$.

5. El-Zawawy H, Mandell BF. Managing gout: how is it different in patients with chronic kidney disease? Cleve Clin J Med 77: 919928, 2010.

6. Schumacher HR Jr, Becker MA, Wortmann RL, et al. Effects of febxostat versus allopurinol and placebo in reducing serum urate in subjects with hyperuricemia and gout: A 28-week, Phase III, randomized, double-blind, parallel-group trial. Arthritis Rheum 59: 1540-1548, 2008.

7. Emmerson BT. The management of gout. N Engl J Med 334: 445451, 1996.

8. Becker MA, Schumacher HR, Wortmann RL, et al. Febuxostat compared with allopurinol in patients with hyperuricemia and gout. N Engl J Med 353: 2450-2461, 2005.

9. Jalal DI, Chonchol M, Chen W, Targher G. Uric acid as a target of therapy in CKD. Am J Kidney Dis 61: 134-146, 2013.

10. Keller DL. Gout and chronic kidney disease. Cleve Clin J Med 78: 81-82, 2011.

11. Reinders MK, van Roon EN, Houtman PM, et al. Biochemical effectiveness of allopurinol and allopurinol-probenecid in previously benzbromarone-treated gout patients. Clin Rheumatol 26: 14591465, 2007.

12. Hosoya T, Ichida K, Tabe A, Sakai O. Combined therapy using allopurinol and benzbromarone for gout and hyperuricemia complicated with renal disorder. J Rheumatol 4: 77-90, 1992.

13. Vogt B. Urate oxidase (rasburicase) for treatment of severe tophaceous gout. Nephrol Dial Transplant 20: 431-433, 2005.

14. Mayer MD, Khosravan R, Vernillet L, Wu JT, Joseph-Ridge N, Mulford DJ. Pharmacokinetics and pharmacodynamics of febuxostat, a new non-purine selective inhibitor of xanthine oxidase in subjects with renal impairment. Am J Ther 12: 22-34, 2005.

(C) 2014 The Japanese Society of Internal Medicine http://www.naika.or.jp/imonline/index.html 